\title{
DIRECT COST ASSOCIATED WITH ADVERSE DRUG REACTIONS AMONG HOSPITALISED CHRONIC KIDNEY PATIENTS IN A PUBLIC HEALTHCARE FACILITY IN MALAYSIA: A RETROSPECTIVE 3-YEAR STUDY
}

\author{
MONICA DANIAL ${ }^{1,2^{*}}$, MOHAMED AZMI HASSALI ${ }^{1}$, ONG LOKE MENG ${ }^{3}$, AMER HAYAT \\ KHAN $^{4}$ AND ANN LISA ARULAPPEN ${ }^{5}$ \\ ${ }^{1}$ Discipline of Social and Administrative Pharmacy, School of Pharmaceutical Sciences, \\ Universiti Sains Malaysia, Pulau Pinang, Malaysia \\ ${ }^{2}$ Clinical Research Center (CRC) Hospital Seberang Jaya, Institute for Clinical Research \\ (ICR), Ministry of Health Malaysia, Pulau Pinang, Malaysia \\ ${ }^{3}$ Clinical Research Center Hospital Pulau Pinang, Institute for Clinical Research, Ministry \\ of Health Malaysia, Pulau Pinang, Malaysia \\ ${ }^{4}$ Discipline of Clinical Pharmacy, School of Pharmaceutical Sciences, Universiti Sains \\ Malaysia, Pulau Pinang, Malaysia \\ ${ }^{5}$ Pharmacy Department, Hospital Seberang Jaya, Pulau Pinang, Malaysia
}

Published online: 23 November 2021

To cite this article: MONICA DANIAL, MOHAMED AZMI HASSALI, ONG LOKE MENG, AMER HAYAT KHAN \& ANN LISA ARULAPPEN (2021) Direct cost associated with adverse drug reactions among hospitalised chronic kidney patients in a public healthcare facility in Malaysia: A retrospective 3-year study, Malaysian Journal of Pharmaceutical Sciences, 19(2): 1-21. https://doi.org/10.21315/mjps2021.19.2.1

To link to this article: https://doi.org/10.21315/mjps2021.19.2.1

\begin{abstract}
Adverse reactions which are clinically diverse increases the overall cost of care, as it often results in additional days of hospitalisation, clinical investigations and treatment drugs. Thus, the main objective of this study is to evaluate direct medical costs among chronic kidney disease (CKD) patients who experienced adverse drug reactions (ADRs) during hospitalisation and identification of associated drug classes and clinical symptoms. Individual direct medical costs from the perspective of Ministry of Health (MOH), Malaysia among stages 3-5 CKD patients who experienced ADRs during hospitalisation were evaluated from 2014 till 2016. A higher number of days of hospitalisation (11.5 [4.25-39.25] days), ward and laboratory costs (RM48.50 [0-195.75]) plus drug costs (RM2.05 [0-91.30]) were observed among patients who did not survive ADRs. The highest number of hospitalisations, monitoring and laboratory costs were attributed to anti-arrhythmic drug class (11.0 [4.00-] days; RM326.00 [0-]) and haematological reactions (11.0 [1.00-19.00] days; RM116.80 [ \pm 112.38$])$. Furthermore, the highest treatment drug cost was attributed to anti-platelet (RM104.60 [0-]) and psychiatric reactions (RM17.50 [ \pm 24.13$]$ ). Top five major treatment drug classes contributed to ADRs were anti-infectives ( $n=63$ [39.4\%]), anti-hypertensive
\end{abstract}

*Corresponding author: monica@crc.moh.gov.my; monicadanialjsm@gmail.com

(c) Penerbit Universiti Sains Malaysia, 2021. This work is licensed under the terms of the Creative Commons Attribution (CC BY) (http://creativecommons.org/licenses/by/4.0/). 
$(n=23[14.4 \%])$, analgesic $(n=12[7.5 \%])$, statin $(n=10[6.3 \%])$ and anti-diabetic $(n=8$ [5.0\%]). Antibacterial constitutes the majority of the anti-infectives reactions. Vancomycin ( $n=7[13.7 \%]$ ) tops the most ADRs contributing antibacterial. ADRs experienced during hospitalisation caused prolongation of hospitalisation and its associated investigational and treatment charges. The true value of the cost estimate could be much higher than the calculated value as the indirect costs were not included in the final estimates of this study and as a result of the Malaysian government's waiver policy.

Keywords: Adverse drug reactions, Chronic kidney disease, Direct medical costs, Healthcare facility, Malaysia

\section{INTRODUCTION}

Chronic kidney disease (CKD) is defined according to the presence or absence of kidney damage and level of kidney function. CKD patients have an increased mortality rate and higher risks of adverse events and toxicity from treatments (Webster et al. 2017), stressing that adverse drug reactions (ADRs) are important factors contributing to morbidity and mortality (Khan et al. 2016; Davies et al. 2009).

Management of ADRs can be costly which utilises a considerable amount of financial resources. ADRs are a major concern for morbidity and mortality in the public health sector, resulting in more than 100,000 deaths in the United States with annual treatment costs of USD136 billion (Tan et al. 2016). Globally, many countries use about $15 \%$ to $20 \%$ of their hospital budgets to manage drug-related complications (Bordet et al. 2001). Impact and administration of ADRs are complex and can cost up to USD30.1 billion annually in the United States. ADRs further increases treatment costs due to the prolongation of hospitalisation and additional clinical investigations. ADRs aggravate treatment cascades when new drugs are prescribed for conditions resulting from another drug usage, which is considered to be an unrecognised ADR. The cost per preventable ADRs was higher than for non-preventable ADRs for ADRs that require hospitalisation (Bates et al. 1997). Another in-patient study reported that the cost per ADR was USD2,262. Hospitalised ADR patients' costs, fluctuate depending on the hospital and wards (Cullen et al. 1997). ADRs occurrence during hospitalisation represents an increase of $9 \%$ in the length of stay and a $20 \%$ increase in the cost of care for bed usage, laboratory and treatment (Khan et al. 2013).

Thus, the main aim of this study is to evaluate the individual direct medical costs among stages 3-5 CKD patients who experienced ADRs during hospitalisation from the perspective of the Ministry of Health (MOH) Malaysia and identification of associated drug classes that contributed to ADRs and its associated clinical symptoms. This is the first study in Malaysia to estimate the direct cost of treating ADRs among hospitalised CKD patients in a general healthcare facility.

\section{METHODS}

\section{Study Design and Patients}

This is a retrospective observational study conducted in General Hospital Pulau Pinang, the second-largest General Hospital in Malaysia. A total of 1,070 medical records of CKD patients who experienced ADRs from various wards for 3 years

Malay J Pharm Sci, Vol. 19, No. 2 (2021): 1-21 
(1 January 2014 and 31 December 2016) were screened for this study. CKD patients in stages 3-5 (estimated glomerular filtration rate [eGFR] $<60 \mathrm{~mL} / \mathrm{min} / 1.73 \mathrm{~m}^{2}$ ) with stable serum creatinine $(\mathrm{sCr})$ values during the initial days of admission and experienced ADRs during hospitalisation were the primary inclusion criteria of this study. The $\mathrm{sCr}$ value obtained during the first day of admission were used to estimate the glomerular filtration rate (GFR). Additional inclusion criteria were patients aged $\geq 18$ years old and admitted for more than $24 \mathrm{~h}$. Medical records that were dubious and incomplete and ward admission due to ADRs or acute kidney injury (AKI) were excluded from this study. Only 160 patients were selected after subsequent screening and identification of records that met the inclusion and exclusion criteria. From the total number of the patient records finally selected, 132 patients survived and 28 patients did not survive from ADRs during hospitalisation. More detailed study methods outlined in this manuscript have been described extensively in our earlier published research articles (Danial et al. 2018; 2019). Before study commencement, ethical approval was obtained from the Medical Research and Ethics Committee (MREC), $\mathrm{MOH}$ Malaysia.

\section{DESCRIPTION OF STUDY OUTCOME}

Individual direct medical costs from the perspective of the $\mathrm{MOH}$ Malaysia, among stages 3-5 CKD patients who experienced ADRs during hospitalisation and identification of associated drug classes and clinical symptoms were the main outcome evaluated in this study.

\section{DATA COLLECTION}

Reported drugs that caused ADRs and the affected organs due to ADRs were recorded from the ADRs reporting form acquired from Drug Information Unit General Hospital Pulau Pinang. The reported ADRs and the affected organs were cross-checked again for any discrepancies in the patients' medical reports. Once, no discrepancies observed in both documents, the drugs and the affected organs were classified into major drug classes and major symptom organ classes.

In this study, only the cost incurred during ADRs were considered. The duration ADRs for each patient were captured from the ADRs reporting form. Direct medical costs associated with ADRs during hospitalisation such as extra days of monitoring and laboratory costs plus treatment drug costs incurred were acquired from the hospital's revenue department database. Monitoring and laboratory costs included incurred ward charges, inpatient charges, laboratory tests charges, ultrasound tests performed and haemodialysis performed. Treatment drug costs were the price of drugs used in treating ADRs. The cost of treatment was considered to be zero for ADRs requiring discontinuation of the suspected drugs. The costs of all these were included in calculating the costs incurred by the individual patient when treating ADRs requiring additional medications, treatments, or laboratory tests. All the cost calculation in this study was done in Ringgit Malaysia (RM).

\section{MINISTRY OF HEALTH, MALAYSIA HEALTHCARE POLICIES}

In Malaysia, at all governments hospitals and clinics waivers of charges and/or a special discount of the hospital's bill are granted for patients infected with all types of infectious diseases or illness, for those who are earning below RM300 per month, the holder of 
Social Welfare or National Islamic Council card, disabled person, students and government servants ( $\mathrm{MOH} 2017 \mathrm{~b}$ ). Also, there is a $50 \%$ reduction with maximum charges of RM250 for each admission for the senior citizens aged 60 years old and above (MOH 2012). Furthermore, most of the monitoring, laboratory and drug costs charges were already at a subsidised rate by the Malaysian government (MOH 2017a). Most of the eligible patients included in this study were over 60 years old and were either waived or given a discount on their total hospital bill. The cost of treatment was considered to be zero for waived payments.

\section{STATISTICAL ANALYSIS}

Major symptom organ classes listed based on major drug classes were presented as frequency $(n)$ and percentage (\%). Patients with multiple ADRs were considered only once during cost analysis. Normally distributed data were reported as mean $\pm \mathrm{SD}$. Non-normally distributed data were reported as median (first quartile [Q1]-third quartile [Q3]). Categorical variables were presented as frequency $(n)$ and percentage (\%). Two-sided $p$-values of less than 0.05 were considered to be statistically significant. Statistical analysis was performed by using IBM SPSS version 22 (SPSS Inc., Chicago, IL).

\section{RESULTS}

Only 160 patients' records were included in this study after the subsequent screening of 1,070 patients records for the duration of 3 years (2014-2016). From the total number of 160 patient records finally selected, 132 patients survived and 28 patients did not survive ADRs during hospitalisation. In this study, patients had experienced more than one ADR events. Patients' demographic characteristics and univariate analysis results performed between survived and did not survive have been reported in our earlier publication (Danial et al. 2019). The comparison was made between patients who survived ADRs and did not survive ADRs in terms of extra days of hospitalisation, estimated ward and laboratory costs plus treatment drug cost. For patients survived ADRs, extra days of hospitalisation, estimated ward and laboratory costs plus treatment drug cost were $2(1.00-5.00)$ days; RM17.50 (0-43.75); RM1.50 (0-20.20) and 11.5 (4.25-39.25) days; RM48.50 (0-195.75); RM2.05 (0-91.30) for patients did not survive ADRs (Table 1).

Table 1: Comparison of patients characteristics according to survivability due to ADR events.

\begin{tabular}{lll}
\hline & \multicolumn{2}{c}{ Number $(\%)$ of patients } \\
\cline { 2 - 3 } Characteristics & Alive $(\boldsymbol{n}=\mathbf{1 3 2})$ & Dead $(\boldsymbol{n}=\mathbf{2 8})$ \\
\hline Demographics & & \\
Age (years old) & & \\
$\leq 49$ & $34(21.3)$ & $7(4.4)$ \\
$50-59$ & $32(20.0)$ & $8(5.0)$ \\
$\geq 60$ & $66(41.3)$ & $13(8.1)$ \\
\hline & & (continued on next page)
\end{tabular}

Malay J Pharm Sci, Vol. 19, No. 2 (2021): 1-21 
Table 1: (continued)

\begin{tabular}{lll}
\hline & \multicolumn{2}{c}{ Number (\%) of patients } \\
\cline { 2 - 3 } Characteristics & Alive $(\boldsymbol{n}=\mathbf{1 3 2})$ & Dead $(\boldsymbol{n}=\mathbf{2 8})$ \\
\hline Gender & $77(48.1)$ & $15(9.4)$ \\
$\quad$ Male & $55(34.4)$ & $13(8.1)$ \\
$\quad$ Female & & \\
Ethnicity & $44(27.5)$ & $8(5.0)$ \\
$\quad$ Malay & $57(35.6)$ & $11(6.9)$ \\
$\quad$ Chinese & $29(18.1)$ & $9(5.6)$ \\
$\quad$ Indian & $2(1.3)$ & $0(0)$ \\
$\quad \begin{array}{l}\text { Others } \\
\text { Renal replacement therapy }\end{array}$ & & $9(5.6)$ \\
$\quad \begin{array}{l}\text { Haemodialysis } \\
\quad \text { Peritoneal dialysis }\end{array}$ & $10(6.3)$ & $2(1.3)$ \\
$\quad \begin{array}{l}\text { Conservative } \\
\text { management }\end{array}$ & $70(43.8)$ & $17(10.6)$ \\
$\begin{array}{l}\text { Extra days of hospitalisation } \\
\text { (days), median (Q1-Q3) }\end{array}$ & $2.0(1.00-5.00)$ & $11.5(4.25-39.25)$ \\
$\begin{array}{l}\text { Monitoring and laboratory } \\
\text { costs (RM), median (Q1-Q3) }\end{array}$ & $17.5(0-43.75)$ & $48.5(0-195.75)$ \\
$\begin{array}{l}\text { Drug costs (RM), } \\
\text { median (Q1-Q3) }\end{array}$ & $1.5(0-20.20)$ & $2.05(0-91.30)$ \\
\hline
\end{tabular}

Extra days of hospitalisation and cost associated with ADRs by symptom organ class and major drug class were illustrated in Table 2 and Table 3. The top five major symptom organ class and major drug class that resulted in extension hospitalisation days were haematological reactions $11.0(1.00-19.00)$ days, electrolyte reactions $5.5(3.25-13.50)$ days, hepatic reactions 5.0 (1.75-13.50) days, psychiatric reactions $5.0(3.00-6.00)$ days, renal reactions $4.5(2.00-14.75)$ days and anti-arrhythmic 11.0 (4.00-) days, anti-psychotics 7.5 (4.00-) days, statin 6.5 (2.50-13.50) days, anti-inflammatory 4.0 (3.00-9.00) days, antiinfective $3.0(1.00-11.00)$ days, respectively.

The top five highest estimated monitoring and laboratory cost from symptom organ class and major drug class were observed among haematological reactions RM116.80 ( \pm 112.38), electrolyte reactions RM44.00 (24.5-143.00), immunological reactions RM35.50 (12.75-69.50), psychiatric reactions RM30.00 (0-151.00), renal reactions RM29.00 (0-57.75), skin reactions RM29.00 (0-90.00) and anti-arrhythmic RM326.00 (0-), antipsychotics RM92.00 (30.0-), anti-gout RM36.00 (27.00-173.00), anti-diabetic RM30.90 $( \pm 32.80)$ and diuretic RM26.00 (0-), respectively.

Drug costs to treat ADRs based on related symptom organ class reactions and major drug class based on top five incurred cost were psychiatric reactions RM17.50 ( \pm 24.13), electrolyte reactions RM6.60 (0-36.79), immunological reactions RM5.70 
(0-36.89), gastrointestinal reactions RM5.30 (0-33.60), neurological reactions RM4.90 $(0-48.00)$ and anti-platelets RM104.60 (0-), immunosuppressant RM45.30 ( \pm 45.64$)$, antiarrhythmic RM20.40 (0-), analgesic RM16.60 ( \pm 16.57) and anti-diabetic RM5.10 (0.0312.68), respectively.

Table 2: Cost associated with ADRs by drug class and symptom organ class among hospitalised CKD patients. All cost are calculated in RM.

\begin{tabular}{lllll}
\hline Major drug class & $\begin{array}{l}\text { Extra days of } \\
\text { hospitalisation } \\
\text { (days), median } \\
\text { (Q1-Q3) }\end{array}$ & $\begin{array}{l}\text { Monitoring and } \\
\text { laboratory costs } \\
\text { (RM), median } \\
\text { (Q1-Q3) }\end{array}$ & $\begin{array}{l}\text { Drug costs } \\
\text { (RM), median } \\
\text { (Q1-Q3) }\end{array}$ & P-value \\
\hline Anti-arrhythmic & $11.0(4.00-)$ & $326.0(0-)$ & $20.4(0-)$ & 0.120 \\
Anti-psychotics & $7.5(4.00-)$ & $92.0(30.0-)$ & nil & \\
Statin & $6.5(2.50-13.50)$ & $0(0-11.25)$ & $0.4(0-4.60)$ \\
Anti-inflammatory & $4.0(3.00-9.00)$ & $23.4( \pm 23.19)$ & $0(0-54.88)$ \\
Anti-infective & $3.0(1.00-11.00)$ & $16.0(0-96.00)$ & $3.6(0-34.05)$ \\
Anti-diabetic & $2.5(19.25,1.25)$ & $30.9( \pm 32.80)^{*}$ & $5.1(0.03-12.68)$ \\
Anti-gout & $2.0(1.00-15.75)$ & $36.0(27.00-173.00)$ & $0(0-9.65)$ \\
Anti-hypertensive & $2.0(1.00-8.00)$ & $22.0(12.00-48.00)$ & $0.3(0-8.55)$ \\
Anti-platelets & $1.50(1.0-)$ & nil & $104.6(0-)$ \\
Analgesic & $1.0(1.00-2.50)$ & $23.5(0-33.50)$ & $16.6( \pm 16.57)^{*}$ & \\
Diuretic & $1.0(1.0-)$ & $26.0(0-)$ & 0 \\
Immunosuppressant & $1.0(1.00-4.50)$ & $21.0(12.00-50.50)$ & $45.3( \pm 45.64)^{*}$ & \\
Anti-angina & nil & 0 & nil \\
Others & $2.0(1.00-5.50)$ & $50.0(0-93.50)$ & $0.5(0-23.55)$ & \\
\hline
\end{tabular}

Note: ${ }^{*}$ mean $( \pm \mathrm{SD})$

Table 3: Cost associated with ADRs by symptom organ class among hospitalised CKD patients. All cost are calculated in RM.

\begin{tabular}{lllll}
\hline Symptom organ class & $\begin{array}{l}\text { Extra days of } \\
\text { hospitalisation } \\
\text { (days), median } \\
\text { (Q1-Q3) }\end{array}$ & $\begin{array}{l}\text { Monitoring and } \\
\text { laboratory costs } \\
\text { (RM), median } \\
(\mathbf{Q 1}-\mathbf{Q} 3)\end{array}$ & $\begin{array}{l}\text { Drug costs } \\
\text { (RM), median } \\
(\mathbf{Q 1}-\mathbf{Q})\end{array}$ & $\boldsymbol{P}$-value \\
\hline Haematological & $11.0(1.00-19.00)$ & $116.8( \pm 112.38)^{*}$ & $0(0-14.70)$ & 0.088 \\
Electrolyte & $5.5(3.25-13.50)$ & $44.0(24.5-143.00)$ & $6.6(0-36.79)$ & 0.685 \\
Hepatic & $5.0(1.75-13.50)$ & $0(0-48.00)$ & $0.4(0-8.48)$ & 0.685 \\
Psychiatric & $5.0(3.00-6.00)$ & $30.0(0-151.00)$ & $17.5( \pm 24.13)^{*}$ & 0.881 \\
\hline
\end{tabular}

(continued on next page) 
Table 3: (continued)

\begin{tabular}{lllll}
\hline Symptom organ class & $\begin{array}{l}\text { Extra days of } \\
\text { hospitalisation } \\
\text { (days), median } \\
\text { (Q1-Q3) }\end{array}$ & $\begin{array}{l}\text { Monitoring and } \\
\text { laboratory costs } \\
\text { (RM), median } \\
\text { (Q1-Q3) }\end{array}$ & $\begin{array}{l}\text { Drug costs } \\
\text { (RM), median } \\
\text { (Q1-Q3) }\end{array}$ & P-value \\
\hline Renal & $4.5(2.00-14.75)$ & $29.0(0-57.75)$ & $0(0-33.60)$ & 0.755 \\
Immunological & $2.5(1.00-6.50)$ & $35.5(12.75-69.50)$ & $5.7(0-36.89)$ & 0.116 \\
Skin & $2.0(1.00-8.00)$ & $29.0(0-90.00)$ & $2.2(0-18.60)$ & 0.689 \\
Gastrointestinal & $2.0(1.00-3.00)$ & $20.0(0-39.0)$ & $5.3(0-33.60)$ & 0.208 \\
Neurological & $2.0(1.00-6.00)$ & $22.0(0-48.00)$ & $4.9(0-48.00)$ & 0.932 \\
Others & $1.5(1.00-4.00)$ & $16.0(0-49.00)$ & $4.0(0-31.04)$ & 0.980 \\
\hline
\end{tabular}

Note: *mean ( \pm SD)

The major drug classes contributed to ADRs for the duration of 3 years were antiinfective, anti-hypertensive, analgesic, statins, anti-diabetic, anti-gout, anti-inflammatory, immunosuppressant, anti-angina, anti- arrhythmic, diuretic, anti-platelets, anti-psychotics and others (Table 4 ). The top five major drug classes contributed to ADRs were anti-infectives ( $n=63[39.4 \%])$, anti-hypertensive $(n=23[14.4 \%])$, analgesic $(n=12[7.5 \%])$, statin $(n=10[6.3 \%])$ and anti-diabetic $(n=8[5.0 \%])$ medications. Anti-infectives adversely causes renal reactions $(n=34$ [21.5\%]), gastrointestinal reactions $(n=31[19.6 \%])$, neurological reactions $(n=27[17.1 \%])$ and skin reactions $(n=23$ [14.6\%]). Anti-hypertensive drug class primarily caused renal reactions $(n=16$ [30.2\%]), skin reactions $(n=12$ [22.6\%]), neurological reactions $(n=6[11.3 \%])$ and hepatic reactions $(n=5[9.4 \%])$. On the other hand, analgesic drug class $(n=12[7.5 \%])$ primarily caused skin reactions $(n=14[51.9 \%])$, gastrointestinal reactions $(n=5[18.5 \%])$ and immunological reactions $(n=3[11.1 \%])$. Statin drug class $(n=10[6.3 \%])$ caused skin reactions $(n=10$ [58.8\%]), immunological reactions $(n=3[17.6 \%])$ and haematological reactions $(n=1[5.9 \%])$. Anti-diabetic medications $(n=8[5.0 \%])$ triggered renal reactions $(n=10[25.0 \%])$, neurological reactions $(n=8$ [20.0\%]), skin reactions $(n=6[15.0 \%])$ and gastrointestinal reactions $(n=6[15.0 \%])$.

Majority of the ADRs were contributed by anti-infectives were caused by antibacterial (Table 5). Vancomycin ( $n=7$ [13.7\%]) tops the five most adverse reaction contributing antibacterial. Vancomycin primarily triggered skin reactions $(n=4[33.3 \%])$, neurological reactions $(n=2[16.7 \%])$ and electrolyte imbalances reactions $(n=2[16.7 \%])$. The antiinfective anti-fungal drug amphotericin B causes neurological reactions $(n=7$ [31.8\%]), renal reactions $(n=6[27.3 \%])$, hepatological reactions $(n=2[9.1 \%])$ and skin reactions $(n=2[9.1 \%])$. The anti-viral drug acyclovir primarily triggers skin reactions $(n=3[27.3 \%])$, renal reactions $(n=3[27.3 \%])$, neurological reactions $(n=2[18.2 \%])$, immunological reactions $(n=2[18.2 \%])$ and electrolyte imbalances reactions $(n=1[9.1 \%])$.

The majority of anti-hypertensive drug effects were caused by drug perindopril. Perindopril primarily triggers renal reactions $(n=10[34.5 \%])$, neurological reactions $(n=4[13.8 \%])$, hepatic reactions $(n=4[13.8 \%])$, skin reactions $(n=3[10.3 \%])$ and gastrointestinal reactions ( $n=3[10.3 \%])$. The third majority of drug classes that contributed to adverse reactions were analgesics. Analgesics such as aspirin, diclofenac and tramal which were reported in this study triggered mostly skin, gastrointestinal and immunological reactions. Simvastatin, atorvastatin and lovastatin were the statins which contributed to adverse reactions in this study. The two types of anti-diabetic drugs attributed to adverse reactions were metformin and gemfibrozil. Metformin accounted for the majority of adverse 
effects by the anti-diabetic drug class. Metformin affects primarily caused renal $(n=10$ [27.0\%]), neurological $(n=8[21.6 \%])$, skin $(n=6$ [16.2\%]), gastrointestinal $(n=4[10.8 \%])$ and immunological $(n=3[8.1 \%])$ reactions (Table 6).

Table 4: Major drug class related ADR frequency and most common symptoms according to organ class.

\begin{tabular}{|c|c|c|c|}
\hline Major drug class & Patients, $n(\%)$ & Symptom organ class & Frequency of ADRs, $n(\%)$ \\
\hline \multirow[t]{10}{*}{ Anti-infective } & $63(39.4)$ & Renal & $34(21.5)$ \\
\hline & & Gastrointestinal & $31(19.6)$ \\
\hline & & Neurological & $27(17.1)$ \\
\hline & & Skin & $23(14.6)$ \\
\hline & & Electrolyte imbalances & $9(5.7)$ \\
\hline & & Haematological & $8(5.1)$ \\
\hline & & Hepatic & $6(3.8)$ \\
\hline & & Immunological & $4(2.5)$ \\
\hline & & Psychiatric & $3(1.9)$ \\
\hline & & Others & $13(8.2)$ \\
\hline \multirow[t]{10}{*}{ Anti-hypertensive } & $23(14.4)$ & Renal & $16(30.2)$ \\
\hline & & Skin & $12(22.6)$ \\
\hline & & Neurological & $6(11.3)$ \\
\hline & & Hepatic & $5(9.4)$ \\
\hline & & Gastrointestinal & $3(5.7)$ \\
\hline & & Immunological & $3(5.7)$ \\
\hline & & Electrolyte imbalances & $1(1.9)$ \\
\hline & & Haematological & $1(1.9)$ \\
\hline & & Psychiatric & $1(1.9)$ \\
\hline & & Others & $5(9.4)$ \\
\hline \multirow[t]{6}{*}{ Analgesic } & $12(7.5)$ & Skin & $14(51.9)$ \\
\hline & & Gastrointestinal & $5(18.5)$ \\
\hline & & Immunological & $3(11.1)$ \\
\hline & & Electrolyte imbalances & $1(3.7)$ \\
\hline & & Renal & $1(3.7)$ \\
\hline & & Others & $3(11.1)$ \\
\hline
\end{tabular}


Table 4: (continued)

\begin{tabular}{|c|c|c|c|}
\hline Major drug class & Patients, $n(\%)$ & Symptom organ class & Frequency of ADRs, $n$ (\%) \\
\hline \multirow[t]{4}{*}{ Statins } & $10(6.3)$ & Skin & $10(58.8)$ \\
\hline & & Immunological & $3(17.6)$ \\
\hline & & Haematological & $1(5.9)$ \\
\hline & & Others & $3(17.6)$ \\
\hline \multirow[t]{6}{*}{ Anti-diabetic } & $8(5.0)$ & Renal & $10(25.0)$ \\
\hline & & Neurological & $8(20.0)$ \\
\hline & & Skin & $6(15.0)$ \\
\hline & & Gastrointestinal & $6(15.0)$ \\
\hline & & Immunological & $3(7.5)$ \\
\hline & & Others & $7(17.5)$ \\
\hline \multirow[t]{7}{*}{ Anti-gout } & $6(3.8)$ & Immunological & $5(25.0)$ \\
\hline & & Neurological & $4(20.0)$ \\
\hline & & Gastrointestinal & $3(15.0)$ \\
\hline & & Skin & $3(15.0)$ \\
\hline & & Renal & $1(5.0)$ \\
\hline & & Hepatic & $1(5.0)$ \\
\hline & & Others & $3(15.0)$ \\
\hline \multirow[t]{6}{*}{ Anti-inflammatory } & $5(3.1)$ & Renal & $5(35.7)$ \\
\hline & & Skin & $3(21.4)$ \\
\hline & & Gastrointestinal & $2(14.3)$ \\
\hline & & Neurological & $1(7.1)$ \\
\hline & & Hepatic & $1(7.1)$ \\
\hline & & Electrolyte imbalances & $2(14.3)$ \\
\hline \multirow[t]{3}{*}{ Immunosuppressant } & $5(3.1)$ & Renal & $4(44.4)$ \\
\hline & & Skin & $3(33.3)$ \\
\hline & & Others & $2(22.2)$ \\
\hline \multirow[t]{6}{*}{ Anti-angina } & $4(2.5)$ & Gastrointestinal & $6(35.3)$ \\
\hline & & Skin & $5(29.4)$ \\
\hline & & Immunological & $2(11.8)$ \\
\hline & & Neurological & $2(11.8)$ \\
\hline & & Renal & $1(5.9)$ \\
\hline & & Hepatic & $1(5.9)$ \\
\hline
\end{tabular}

Malay J Pharm Sci, Vol. 19, No. 2 (2021): 1-21 
Table 4: (continued)

\begin{tabular}{|c|c|c|c|}
\hline Major drug class & Patients, $n(\%)$ & Symptom organ class & Frequency of ADRs, $n(\%)$ \\
\hline \multirow[t]{4}{*}{ Anti-arrhythmic } & $3(1.9)$ & Renal & $5(38.5)$ \\
\hline & & Immunological & $4(30.8)$ \\
\hline & & Gastrointestinal & $2(15.4)$ \\
\hline & & Neurological & $2(15.4)$ \\
\hline \multirow[t]{3}{*}{ Diuretic } & $3(1.9)$ & Gastrointestinal & $2(50.0)$ \\
\hline & & Hepatic & $1(25.0)$ \\
\hline & & Others & $1(25.0)$ \\
\hline \multirow[t]{3}{*}{ Anti-platelets } & $2(1.3)$ & Renal & $4(50.0)$ \\
\hline & & Gastrointestinal & $3(37.5)$ \\
\hline & & Electrolyte imbalances & $1(12.5)$ \\
\hline \multirow[t]{2}{*}{ Anti-psychotics } & $2(1.3)$ & Gastrointestinal & $2(50.0)$ \\
\hline & & Neurological & $2(50.0)$ \\
\hline \multirow[t]{8}{*}{ Others } & $14(8.8)$ & Skin & $6(30.0)$ \\
\hline & & Neurological & $4(20.0)$ \\
\hline & & Gastrointestinal & $3(15.0)$ \\
\hline & & Haematological & $2(10.0)$ \\
\hline & & Hepatic & $1(5.0)$ \\
\hline & & Electrolyte imbalances & $1(5.0)$ \\
\hline & & Psychiatric & $1(5.0)$ \\
\hline & & Others & $2(10.0)$ \\
\hline
\end{tabular}

Table 5: Anti-infectives related ADR frequency and most common symptoms according to organ class.

Major drug class Patients, $n(\%)$ Symptom organ class Frequency of ADRs, $n(\%)$

Antibacterial

\begin{tabular}{|c|c|c|c|}
\hline \multirow[t]{6}{*}{ Vancomycin } & \multirow[t]{6}{*}{$7(13.7)$} & Skin & $4(33.3)$ \\
\hline & & Neurological & $2(16.7)$ \\
\hline & & Electrolyte imbalances & $2(16.7)$ \\
\hline & & Gastrointestinal & $1(8.3)$ \\
\hline & & Immunological & $1(8.3)$ \\
\hline & & Others & $2(16.7)$ \\
\hline
\end{tabular}

(continued on next page)

Malay J Pharm Sci, Vol. 19, No. 2 (2021): 1-21 
Table 5: (continued)

\begin{tabular}{|c|c|c|c|}
\hline Major drug class & Patients, $n(\%)$ & Symptom organ class & Frequency of ADRs, $n(\%)$ \\
\hline \multirow[t]{7}{*}{ Cefazolin } & $5(9.8)$ & Electrolyte imbalances & $4(28.6)$ \\
\hline & & Skin & $4(28.6)$ \\
\hline & & Renal & $1(7.1)$ \\
\hline & & Gastrointestinal & $1(7.1)$ \\
\hline & & Hepatic & $1(7.1)$ \\
\hline & & Psychiatric & $1(7.1)$ \\
\hline & & Others & $2(14.3)$ \\
\hline \multirow[t]{5}{*}{ Unasyn } & $4(7.8)$ & Electrolyte imbalances & $5(38.5)$ \\
\hline & & Neurological & $4(30.8)$ \\
\hline & & Immunological & $2(15.4)$ \\
\hline & & Renal & $1(7.7)$ \\
\hline & & Others & $1(7.7)$ \\
\hline \multirow[t]{3}{*}{ Augmentin } & $3(5.9)$ & Neurological & $7(63.6)$ \\
\hline & & Electrolyte imbalances & $2(18.2)$ \\
\hline & & Others & $2(18.2)$ \\
\hline \multirow[t]{3}{*}{ Cefepime } & $3(5.9)$ & Hepatic & $2(50.0)$ \\
\hline & & Immunological & $1(25.0)$ \\
\hline & & Haematological & $1(25.0)$ \\
\hline \multirow[t]{4}{*}{ Ceftazidime } & $3(5.9)$ & Skin & $3(37.5)$ \\
\hline & & Renal & $2(25.0)$ \\
\hline & & Hepatic & $2(25.0)$ \\
\hline & & Neurological & $1(12.5)$ \\
\hline \multirow[t]{4}{*}{ Meropenem } & $3(5.9)$ & Hepatic & $2(33.3)$ \\
\hline & & Renal & $2(33.3)$ \\
\hline & & Neurological & $1(16.7)$ \\
\hline & & Others & $1(16.7)$ \\
\hline \multirow[t]{2}{*}{ Ertapenem } & $2(3.9)$ & Electrolyte imbalances & $3(60.0)$ \\
\hline & & Renal & $2(40.0)$ \\
\hline \multirow[t]{2}{*}{ Erythromycin } & $2(3.9)$ & Renal & $1(50.0)$ \\
\hline & & Psychiatric & $1(50.0)$ \\
\hline
\end{tabular}

Malay J Pharm Sci, Vol. 19, No. 2 (2021): 1-21 
Table 5: (continued)

\begin{tabular}{|c|c|c|c|}
\hline Major drug class & Patients, $n(\%)$ & Symptom organ class & Frequency of ADRs, $n(\%)$ \\
\hline \multirow[t]{2}{*}{ Imipenem } & $2(3.9)$ & Psychiatric & $3(60.0)$ \\
\hline & & Renal & $2(40.0)$ \\
\hline \multirow[t]{2}{*}{ Linezolid } & $2(3.9)$ & Skin & $3(60.0)$ \\
\hline & & Gastrointestinal & $2(40.0)$ \\
\hline \multirow[t]{2}{*}{ Sulperazone } & $2(3.9)$ & Skin & $3(75.0)$ \\
\hline & & Others & $1(25.0)$ \\
\hline \multirow[t]{4}{*}{ Amoxycillin } & $2(3.9)$ & Electrolyte imbalances & $2(40.0)$ \\
\hline & & Renal & $1(20.0)$ \\
\hline & & Immunological & $1(20.0)$ \\
\hline & & Others & $1(20.0)$ \\
\hline \multirow[t]{7}{*}{ Others } & $11(21.6)$ & Renal & $8(34.8)$ \\
\hline & & Electrolyte imbalances & $6(26.1)$ \\
\hline & & Neurological & $3(13.0)$ \\
\hline & & Skin & $1(4.3)$ \\
\hline & & Immunological & $1(4.3)$ \\
\hline & & Psychiatric & $1(4.3)$ \\
\hline & & Others & $3(13.0)$ \\
\hline \multicolumn{4}{|l|}{ Anti-fungal } \\
\hline \multirow[t]{8}{*}{ Amphotericin B } & $7(87.5)$ & Neurological & $7(31.8)$ \\
\hline & & Renal & $6(27.3)$ \\
\hline & & Haematological & $2(9.1)$ \\
\hline & & Skin & $2(9.1)$ \\
\hline & & Immunological & $1(4.5)$ \\
\hline & & Hepatic & $1(4.5)$ \\
\hline & & Electrolyte imbalances & $1(4.5)$ \\
\hline & & Others & $2(9.1)$ \\
\hline \multirow[t]{3}{*}{ Fluconazole } & $1(12.5)$ & Neurological & $3(50.0)$ \\
\hline & & Renal & $2(33.3)$ \\
\hline & & Electrolyte imbalances & $1(16.7)$ \\
\hline
\end{tabular}

Malay J Pharm Sci, Vol. 19, No. 2 (2021): 1-21 
Table 5: (continued)

\begin{tabular}{llll}
\hline Major drug class & Patients, $\boldsymbol{n}(\%)$ & Symptom organ class & Frequency of ADRs, $\boldsymbol{n}(\%)$ \\
\hline Anti-viral & & & \\
Acyclovir & $3(75.0)$ & Skin & $3(27.3)$ \\
& & Renal & $3(27.3)$ \\
& & Neurological & $2(18.2)$ \\
& & Immunological & $2(18.2)$ \\
& & Electrolyte imbalances & $1(9.1)$ \\
Lamivudine & $1(25.0)$ & Renal & $4(100.0)$ \\
Abacavir & & & \\
\hline
\end{tabular}

Table 6: Anti-hypertensive, analgesic, statins and anti-diabetic related frequency and most common symptoms according to organ class.

\begin{tabular}{|c|c|c|c|}
\hline Major drug class & Patients, $n(\%)$ & Symptom organ class & Frequency of ADRs, $n(\%)$ \\
\hline \multicolumn{4}{|l|}{ Anti-hypertensive } \\
\hline \multirow[t]{8}{*}{ Perindopril } & $14(60.9)$ & Renal & $10(34.5)$ \\
\hline & & Neurological & $4(13.8)$ \\
\hline & & Hepatic & $4(13.8)$ \\
\hline & & Skin & $3(10.3)$ \\
\hline & & Gastrointestinal & $3(10.3)$ \\
\hline & & Immunological & $2(6.9)$ \\
\hline & & Haematological & $1(3.4)$ \\
\hline & & Others & $2(6.9)$ \\
\hline \multirow[t]{4}{*}{ Minoxidil } & $2(8.70)$ & Skin & $3(50.0)$ \\
\hline & & Immunological & $1(16.7)$ \\
\hline & & Neurological & $1(16.7)$ \\
\hline & & Psychiatric & $1(16.7)$ \\
\hline \multirow[t]{2}{*}{ Prazosin } & $2(8.70)$ & Renal & $3(75.0)$ \\
\hline & & Skin & $1(25.0)$ \\
\hline \multirow[t]{4}{*}{ Others } & $5(21.7)$ & Skin & $5(35.7)$ \\
\hline & & Renal & $3(21.4)$ \\
\hline & & Electrolyte imbalances & $1(7.1)$ \\
\hline & & Neurological & $1(7.1)$ \\
\hline
\end{tabular}


Table 6: (continued)

\begin{tabular}{llll}
\hline Major drug class & Patients, $\boldsymbol{n}(\%)$ & Symptom organ class & Frequency of ADRs, $\boldsymbol{n}(\%)$ \\
\hline & Hepatic & $1(7.1)$ \\
& Others & $3(21.4)$
\end{tabular}

\section{Analgesic}

\begin{tabular}{|c|c|c|c|}
\hline \multirow[t]{5}{*}{ Aspirin } & $4(33.3)$ & Skin & $1(20.0)$ \\
\hline & & Gastrointestinal & $1(20.0)$ \\
\hline & & Immunological & $1(20.0)$ \\
\hline & & Renal & $1(20.0)$ \\
\hline & & Others & $1(20.0)$ \\
\hline \multirow[t]{2}{*}{ Diclofenac } & $3(25.0)$ & Skin & $4(80.0)$ \\
\hline & & Electrolyte imbalances & $1(20.0)$ \\
\hline \multirow[t]{4}{*}{ Tramadol } & $3(25.0)$ & Skin reactions & $3(37.5)$ \\
\hline & & Gastrointestinal & $3(37.5)$ \\
\hline & & Immunological & $1(12.5)$ \\
\hline & & Others & $1(12.5)$ \\
\hline \multirow[t]{4}{*}{ Others } & $2(16.7)$ & Skin & $6(66.7)$ \\
\hline & & Gastrointestinal & $1(11.1)$ \\
\hline & & Immunological & $1(11.1)$ \\
\hline & & Others & $1(11.1)$ \\
\hline \multicolumn{4}{|l|}{ Statins } \\
\hline \multirow[t]{4}{*}{ Simvastatin } & $5(50.0)$ & Skin & $8(53.3)$ \\
\hline & & Immunological & $3(20.0)$ \\
\hline & & Haematological & $1(6.7)$ \\
\hline & & Others & $3(20.0)$ \\
\hline \multirow[t]{4}{*}{ Atorvastatin } & $3(30.0)$ & Skin & $2(25.0)$ \\
\hline & & Gastrointestinal & $2(25.0)$ \\
\hline & & Neurological & $1(12.5)$ \\
\hline & & Others & $3(37.5)$ \\
\hline \multirow[t]{3}{*}{ Lovastatin } & $2(20.0)$ & Immunological & $2(33.3)$ \\
\hline & & Neurological & $2(33.3)$ \\
\hline & & Psychiatric & $2(33.3)$ \\
\hline
\end{tabular}


Table 6: (continued)

\begin{tabular}{llll}
\hline Major drug class & Patients, $\boldsymbol{n}(\%)$ & Symptom organ class & Frequency of ADRs, $\boldsymbol{n}(\%)$ \\
\hline Anti-diabetic & & & \\
Metformin & $7(87.5)$ & Renal & $10(27.0)$ \\
& & Neurological & $8(21.6)$ \\
& & Skin & $6(16.2)$ \\
& & Gastrointestinal & $4(10.8)$ \\
& & Immunological & $3(8.1)$ \\
& & Others & $6(16.2)$ \\
Gemfibrozil & \multirow{2}{*}{$1(12.5)$} & Gastrointestinal & $4(10.8)$ \\
& & Others & $1(33.3)$ \\
\hline
\end{tabular}

\section{DISCUSSION}

ADRs contributed to a longer duration of hospitalisation coupled with a higher ward, laboratory and drug costs in this study. Our findings were supported by previously published studies (Classen et al. 1997; Phillips, Christenfeld and Glynn 1998; Budnitz et al. 2006). The differences in ADRs costing outcomes were influenced by factors such as disease prevalence, culture differences, ethnicity and economic status (Chan et al. 2015; Jung et al. 2017). The top five major drug classes that contributed to ADRs among our study patients were anti-infectives, anti-hypertensive, analgesic, statin and anti-diabetic medications.

Prolongation of hospitalisation days remains the parameter that best reflects the increase in ADRs' direct cost (Bordet et al. 2001). The estimated cost in this study is much lower than data from other published studies (Rottenkolber, Hasford and Stausberg 2012; Bates et al. 1997; Bates et al. 1999; Jha et al. 2001; Dartnell et al. 1996). However, these published studies also reported that the costs reported were very expensive. In their final cost calculations, ADRs-associated risks in ambulatory treatment were also included in the review of cost accountability concerning the origin of these studies (Thurmann 2001). Moreover, the higher cost reported by some previous studies may be due to the severity of the ADRs (Chan et al. 2008). In addition, lower cost estimation in this study may due to waiver policy implemented by the Malaysian government as the majority of the patients in this study were more than 60 years old. Also, since Malaysia has centralised its public sector administration, it is subjected to policy and programmes formulated and financed by its $\mathrm{MOH}$. Also, lower-cost estimation in this study may due to the waiver policy implemented by the Malaysian government as the majority of the patients in this study were more than 60 years old. Also, since Malaysia has centralised its public sector administration, it is subjected to policy and programmes formulated and financed by its $\mathrm{MOH}$. Therefore, patients will only be required to pay minimum fees as low as RM1 when treated at government health facilities which may influence the actual total payable amount (Rosli et al. 2017).

The highest number of days of hospitalisation and the estimated direct cost of monitoring and laboratory costs were associated with haematological reactions from this study. Treating haematological reactions often results in the continuation of hospital care coupled with tough clinical decisions when patients have advanced disease states such 
as kidney disease and alternative treatment options are limited (Ganguli et al. 2015). The advanced state of kidney disease also emerges as an important complication that further complicates the treatment. Moreover, it was reported that hospitalised patients with kidney impairment with haematological reaction possess the highest mortality rate (Salahudeen et al. 2013; de Mendonca et al. 2000). Other associated risk factors were older age, mechanical ventilation and the use of nephrotoxic medications (Lahoti et al. 2010).

In this study, the electrolyte reaction was also one of the important factors that contributed to the prolongations of hospitalisation and higher monitoring, laboratory and drug costs. Instability of $\mathrm{pH}$ even for a short time might lead to a vast array of adverse effects (Dhondup and Qian 2017). The kidneys play a key role in electrolyte and acid-base balance regulation. In the case of kidney insufficiency, electrolyte and acid-base imbalances occur such as hyperkalaemia, metabolic acidosis and hyperphosphatemia which results in severe complications such as muscle loss, bone-mineral disorder, vascular calcification and mortality (Raphael et al. 2011; Kovesdy et al. 2009).

Moreover, another important disorder that contributed to the highest drug treatment cost and moderate prolongation of hospitalisation, monitoring and laboratory cost were psychiatric reactions. Psychiatric reactions such as depression and anxiety are associated with lower quality of life among CKD patients as it is associated with lower treatment adherence, frequent hospitalisations and increased mortality (Kimmel et al. 2019), which directly increases the cost of treatment.

Also, CKD patients with diabetes were significantly associated with higher morbidity and mortality, mainly due to high cardiovascular risk (Grandfils et al. 2013). Metformin is one of the common drugs for type 2 diabetes treatment (American Diabetes Association 2014). Metformin mainly acts by decreasing the production of hepatic glucose, increasing the peripheral absorption of glucose, improving glucose tolerance and lowering fasting and postprandial plasma glucose. Metformin should be prescribed with caution as it is renally excreted and it results in the development of lactic acidosis as the most serious adverse effect (Game 2014).

In addition to the direct financial costs, patients and their caregivers also have several indirect expenses incurred by ADRs, such as missed working days and/or morbidity, such as anxiety caused by the ADR episodes (Wu and Pantaleo 2003). Other related expenses are wages of the medical personnel, disposable goods and medications (Wasserfallen et al. 2001). Prevention efforts can be undertaken by implementing ADR evidence-based surveillance programs in healthcare facilities which may focus on ADR causative drug classes to reduce human consequences and economic costs which effectively leads to cost savings (Dormann et al. 2000; Tan et al. 2016).

\section{CONCLUSION}

To the best of our knowledge, this is the first study in Malaysia to estimate the direct cost of treating ADRs that occurs during hospitalisation among CKD patients in a general healthcare facility. Our finding showed that ADRs experienced during hospitalisation stay caused prolongation of hospitalisation and its' associated investigational and treatment charges. The true value of cost estimation might be far greater than the calculated value as the indirect costs were not included in the final estimation of the present study and due to the waiver policy implemented by the Malaysian government. This study outcome hoped to contribute some preliminary information on the cost of treating ADRs among hospitalised CKD patients in Malaysia for more in-depth future research.

Malay J Pharm Sci, Vol. 19, No. 2 (2021): 1-21 


\section{STUDY LIMITATIONS}

Estimation of the actual hospitalisation cost due to ADRs could not be determined as the indirect cost is not taken into account in this study and the estimation of the direct hospitalisation cost is impaired by waiver policy and subsidisation by the Malaysian government. Furthermore, since the cost was estimated from the perspective of the $\mathrm{MOH}$, the study results may not reflect the cost in private hospitals as drugs are procured at subsidised rates for public hospitals in Malaysia.

\section{ACKNOWLEDGEMENTS}

The authors wish to acknowledge Mr. Soelar Aiman (CRC Sultanah Bahiyah, Alor Setar, Malaysia) for his statistical guidance and feedback. We would like to thank the Director General of Health Malaysia for permission to publish this article.

\section{DECLARATIONS}

\section{Ethical Approval}

The study was carried out after obtaining the approval from the Medical Research \& Ethics Committee (MREC), Ministry of Health Malaysia (MOH). Study approval number: NMRR15-1810-28375(IIR).

The need to obtain consent from study participants was waived as there is no direct interaction with the study participants following the MREC, $\mathrm{MOH}$ regulations.

\section{Availability of Data and Material}

The datasets generated and analysed during the current study are not publicly available due to data confidentiality policy as dictated in the study approval letter by the MREC, $\mathrm{MOH}$ (Ref no: (5) KKM/NIHSEC/P16-25) but are available from the corresponding author on reasonable request.

\section{REFERENCES}

AMERICAN DIABETES ASSOCIATION (2014) Standards of medical care in diabetes 2014. Diabetes Care, 37(Suppl 1): S14-S80. https://doi.org/10.2337/dc14-S014

BATES, D. W., MILLER, E. B., CULLEN, D. J., BURDICK, L., WILLIAMS, L., LAIRD, N. et al. (1999) Patient risk factors for adverse drug events in hospitalized patients. ADE Prevention Study Group, Archives of Internal Medicine, 159(21): 2553-2560. https://doi.org/10.1001/ archinte.159.21.2553

BATES, D. W., SPELL, N., CULLEN, D. J., BURDICK, E., LAIRD, N., PETERSEN, L. A. et al. (1997) The costs of adverse drug events in hospitalized patients. ADE Prevention Study Group, JAMA, 277(4): 307-311. https://doi.org/10.1001/jama.1997.03540280045032 
BORDET, R., GAUTIER, S., LE LOUET, H., DUPUIS, B. \& CARON, J. (2001) Analysis of the direct cost of adverse drug reactions in hospitalized patients, European Journal of Clinical Pharmacology, 56: 935-941. https://doi.org/10.1007/s002280000260

BUDNITZ, D. S., POLLOCK, D. A., WEIDENBACH, K. N., MENDELSOHN, A. B., SCHROEDER, T. J. \& ANNEST, J. L. (2006) National surveillance of emergency department visits for outpatient adverse drug events, JAMA, 296(15): 1858-1866. https:// doi.org/10.1001/jama.296.15.1858

CHAN, A. L., LEE, H. Y., HO, C. H., CHAM, T. M. \& LIN, S. J. (2008) Cost evaluation of adverse drug reactions in hospitalized patients in Taiwan: A prospective, descriptive, observational study, Current Therapeutic Research Clinical and Experimental, 69(2): 118129. https://doi.org/10.1016/j.curtheres.2008.04.005

CHAN, S. L., JIN, S., LOH, M. \& BRUNHAM, L. R. (2015) Progress in understanding the genomic basis for adverse drug reactions: A comprehensive review and focus on the role of ethnicity, Pharmacogenomics, 16(10): 1161-1178. https://doi.org/10.2217/PGS.15.54

CLASSEN, D. C., PESTOTNIK, S. L., EVANS, R. S., LLOYD, J. F. \& BURKE, J. P. (1997) Adverse drug events in hospitalized patients. Excess length of stay, extra costs, and attributable mortality, Obstetrical and Gynecological Survey, 52(5): 291-292. https://doi. org/10.1097/00006254-199705000-00016

CULLEN, D. J., SWEITZER, B. J., BATES, D. W., BURDICK, E. F., EDMONDSON, A. \& LEAPE, L. L. (1997) Preventable adverse drug events in hospitalized patients: A comparative study of intensive care and general care units, Critical Care Medicine, 25(8): 1289-1297. https://doi.org/10.1097/00003246-199708000-00014

DANIAL, M., HASSALI, M. A., ONG, L. M. \& KHAN, A. H. (2018) Survivability of hospitalized chronic kidney disease (CKD) patients with moderate to severe estimated glomerular filtration rate (eGFR) after experiencing adverse drug reactions (ADRs) in a public healthcare center: A retrospective 3-year study, BMC Pharmacology and Toxicology, 19: 52. https:// doi.org/10.1186/s40360-018-0243-0

DANIAL, M., HASSALI, M. A., ONG, L. M. \& KHAN, A. H. (2019) Development of a mortality score to assess risk of adverse drug reactions among hospitalized patients with moderate to severe chronic kidney disease, BMC Pharmacology and Toxicology, 20:1-11. https://doi. org/10.1186/s40360-019-0318-6

DARTNELL, J. G., ANDERSON, R. P., CHOHAN, V., GALBRAITH, K. J., LYON, M. E., NESTOR, P. J. et al. (1996) Hospitalization for adverse events related to drug therapy: Incidence, avoidability and costs, The Medical Journal of Australia, 164(11): 659-662. https://doi.org/10.5694/j.1326-5377.1996.tb122235.x

DAVIES, E. C., GREEN, C. F., TAYLOR, S., WILLIAMSON, P. R., MOTTRAM, D. R. \& PIRMOHAMED, M. (2009) Adverse drug reactions in hospital in-patients: A prospective analysis of 3695 patient-episodes, PLoS One, 4(2): e4439. https://doi.org/10.1371/journal. pone.0004439 
DE MENDONCA, A., VINCENT, J. L., SUTER, P. M., MORENO, R., DEARDEN, N. M., ANTONELLI, M. et al. (2000) Acute renal failure in the ICU: Risk factors and outcome evaluated by the SOFA score. Intensive Care Medicine, 26: 915-921. https://doi. org/10.1007/s001340051281

DHONDUP, T. \& QIAN, Q. (2017) Electrolyte and acid-base disorders in chronic kidney disease and end-stage kidney failure, Blood Purification, 43(1-3): 179-188. https://doi. org/10.1159/000452725

DORMANN, H., MUTH-SELBACH, U., KREBS, S., CRIEGEE-RIECK, M., TEGEDER, I., SCHNEIDER, H. T. et al. (2000) Incidence and costs of adverse drug reactions during hospitalization: Computerised monitoring versus stimulated spontaneous reporting, Drug Safety, 22: 161-168. https://doi.org/10.2165/00002018-200022020-00007

GAME, F. (2014) Novel hypoglycaemic agents: Considerations in patients with chronic kidney disease, Nephron Clinical Practice, 126(1): 14-18. https://doi.org/10.1159/000357680

GANGULI, A., SAWINSKI, D. \& BERNS, J. S. (2015) Kidney diseases associated with hematological cancers. Nature Reviews. Nephrology, 11: 478-490. https://doi.org/10.1038/ nrneph.2015.81

GRANDFILS, N., DETOURNAY, B., ATTALI, C., JOLY, D., SIMON, D., VERGES, B. et al. (2013) Glucose lowering therapeutic strategies for type 2 diabetic patients with chronic kidney disease in primary care setting in France: a cross-sectional study, International Journal of Endocrinology, 2013: 1-6. https://doi.org/10.1155/2013/640632

JHA, A. K., KUPERMAN, G. J., RITTENBERG, E., TEICH, J. M. \& BATES, D. W. (2001) Identifying hospital admissions due to adverse drug events using a computer-based monitor. Pharmacoepidemiology and Drug Safety, 10(2): 113-119. https://doi.org/10.1002/pds.568

JUNG, I. Y., KIM, J. J., LEE, S. J., KIM, J., SEONG, H., JEONG, W. et al. (2017) Antibioticrelated adverse drug reactions at a tertiary care hospital in South Korea, BioMed Research International, 2017: 1-7. https://doi.org/10.1155/2017/4304973

KHAN, L. M., AL-HARTHI, S. E., OSMAN, A. M. M., SATTAR, M. A. A. A. \& ALI, A. S. (2016) Dilemmas of the causality assessment tools in the diagnosis of adverse drug reactions, Saudi Pharmaceutical Journal, 24(4): 485-493. https://doi.org/10.1016/j.jsps.2015.01.010

KHAN, L. M., AL-HARTHI, S. E. \& SAADAH, O. I. (2013)Adverse drug reactions in hospitalized pediatric patients of Saudi Arabian University Hospital and impact of pharmacovigilance in reporting ADR, Saudi Pharmaceutical Journal, 21(3): 261-266. https://doi.org/10.1016/j. jsps.2012.09.004

KIMMEL, P. L., FWU, C.-W., ABBOTT, K. C., MOXEY-MIMS, M. M., MENDLEY, S., NORTON, J. M. et al. (2019) Psychiatric illness and mortality in hospitalized ESKD dialysis patients. Clinical Journal of the American Society of Nephrology, 14(9): 1363-1371. https:// doi.org/10.2215/CJN.14191218 
KOVESDY, C. P., ANDERSON, J. E. \& KALANTAR-ZADEH, K. (2009) Association of serum bicarbonate levels with mortality in patients with non-dialysis-dependent CKD, Nephrology, Dialysis, Transplantation, 24(4): 1232-1237. https://doi.org/10.1093/ndt/gfn633

LAHOTI, A., KANTARJIAN, H., SALAHUDEEN, A. K., RAVANDI, F., CORTES, J. E., FADERL, S. et al. (2010) Predictors and outcome of acute kidney injury in patients with acute myelogenous leukemia or high-risk myelodysplastic syndrome, Cancer, 116(17): 4063-4068. https://doi.org/10.1002/cncr.25306

Ministry of Health (MOH) Malaysia (2012) Pelaksanaan pengecualian caj pendaftaran jabatan pesakit luar pakar sebanyak RM 5.00 dan pengurangan caj sebanyak $50 \%$ bagi pesakit kelas 3 di hospital/klinik Kementerian Kesihatan Malaysia kepada semua pesakit warganegara yang berumur 60 tahun dan ke atas. Putrajaya, Malaysia: Ministry of Health Malaysia.

Ministry of Health (MOH) Malaysia (2017a) Garis panduan pelaksanaan perintah $\mathrm{Fi}$ (Perubatan) (Pindaan) 2017. Putrajaya, Malaysia: Ministry of Health Malaysia.

Ministry of Health (MOH) Malaysia (2017b) Who might be eligible for hospital's bill exemption or discount? [Online]. Putrajaya, Malaysia: Ministry of Health Malaysia. Available at: http:// www.moh.gov.my/english.php/pages/view/160 [Retrieved 2017 Jan 4].

PHILLIPS, D. P., CHRISTENFELD, N. \& GLYNN, L. M. (1998) Increase in US medicationerror deaths between 1983 and 1993, Lancet, 351(9103): 643-644. https://doi.org/10.1016/ S0140-6736(98)24009-8

RAPHAEL, K. L., WEI, G., BAIRD, B. C., GREENE, T. \& BEDDHU, S. (2011) Higher serum bicarbonate levels within the normal range are associated with better survival and renal outcomes in African Americans, Kidney Int, 79(3): 356-362. https://doi.org/10.1038/ ki.2010.388

ROSLI, R., DALI, A. F., AZIZ, N. A., MING, L. C. \& MANAN, M. M. (2017) Reported adverse drug reactions in infants: A nationwide analysis in Malaysia, Frontiers in Pharmacology, 8: 30. https://doi.org/10.3389/fphar.2017.00030

ROTTENKOLBER, D., HASFORD, J. \& STAUSBERG, J. (2012) Costs of adverse drug events in German hospitals - A microcosting study, Value Health, 15(6): 868-875. https:// doi.org/10.1016/j.jval.2012.05.007

SALAHUDEEN, A. K., DOSHI, S. M., PAWAR, T., NOWSHAD, G., LAHOTI, A. \& SHAH, P. (2013) Incidence rate, clinical correlates, and outcomes of AKI in patients admitted to a comprehensive cancer center, Clinical Journal of the Amerian Society of Nephrology, 8(3): 347-354. https://doi.org/10.2215/CJN.03530412

TAN, Y., HU, Y., LIU, X., YIN, Z., CHEN, X. W. \& LIU, M. (2016) Improving drug safety: From adverse drug reaction knowledge discovery to clinical implementation, Methods, 110: 14-25.https://doi.org/10.1016/j.ymeth.2016.07.023

THURMANN, P. A. (2001) Methods and systems to detect adverse drug reactions in hospitals, Drug Safety, 24: 961-968. https://doi.org/10.2165/00002018-200124130-00003

Malay J Pharm Sci, Vol. 19, No. 2 (2021): 1-21 
WASSERFALLEN, J., LIVIO, F., BUCLIN, T., TILLET, L., YERSIN, B. \& BIOLLAZ, J. (2001) Rate, type, and cost of adverse drug reactions in emergency department admissions, European Journal of Internal Medicine, 12(5): 442-447. https://doi.org/10.1016/S09536205(01)00159-5

Webster, A. C., NAGLER, E. V., MORTON, R. L. \& MASSON, P. (2017) Chronic kidney disease, The Lancet, 389(10075): 1238-1252. https://doi.org/10.1016/S01406736(16)32064-5

WU, W. K. \& PANTALEO, N. (2003) Evaluation of outpatient adverse drug reactions leading to hospitalization. American Journal of Health System Pharmacy, 60(3): 253-259. https://doi.org/10.1093/ajhp/60.3.253 